\title{
Suggestions for the Preliminary Definition of a Current Background Profile of Polychlorodibenzodioxin (PCDDs) and Polychlorodibenzofuran (PCDFs) Congeners in Serum Samples
}

\author{
Roberto Miniero*, Elena De Felip and Alessandro di Domenico \\ Chemical Toxicology Unit, Department of the Environment and Primary Intervention, Istituto Superiore di Sanità, Rome, Italy
}

Keywords: Dioxins; Furans; Profile; Human blood; Background

\section{Introduction}

The congener specific polychlorodibenzodioxins (PCDDs) and polychlorodibenzofurans (PCDFs) distribution in human body is a function of bioavailability processes needed to cross the biological membranes. The blood is the destination of major distribution of chemicals into the body. This matrix appears the most important to interpret the concentration levels determined in terms of toxicity or to link them to the interpretation of temporal and spatial trends.

\section{Theoretical and Experimental}

The distribution of PCDD and PCDF congeners in human serum samples may vary according to the source(s) of exposure encountered by the subjects, but food is the main source and the variable levels of these contaminants in human tissues reflect the differing levels of contamination in different foods. In breast milk samples, the congeners from possible different dietary patterns appear to correspond to the widespread combustion profile [1]. However, the precise extent of the aforesaid variation is not known but would be extremely useful when assessing background contamination profiles. These in turn could help in monitoring the efficacy of measures aimed at reducing environmental levels of PCDDs and PCDFs, such as those established in the Stockholm Convention on Persistent Organic Pollutants [2]. There are 17 PCDD and PCDF congeners of toxicological relevance usually determined in human tissues and they exert their toxicity via a common mechanism of action [3]. Their presence in all environmental and human matrices is determined by high resolution gas chromatography coupled with high resolution mass spectrometry (HRGC-HRMS). The database we used comprises a sub-selection of subjects enrolled in a number of biomonitoring research projects completed in different Regions of Italy over the last three years. This preliminary sub-selection was based only on an evaluation of the possible sources of exposure and did not consider the age of subjects as a weighting factor; this will be included in future reports on these researches in this area. All the samples were collected from hypothetical background areas. One hundred matrices (each being a pool of ten subjects) were analyzed. This preliminary investigation was performed using Principal Component Analysis [4].

\section{Results and Discussion}

Table 1 shows the Principal Component (PC) loadings with their variability estimates. The dataset is described by just two Principal Components, suggesting that the potential sources of exposure do not influence greatly the separation of samples. In Table 1 , only one component takes into account the major variance quote $(66.7 \%)$ which confirms that the prevalent congeners mark this variance quote of the dataset. Therefore, these congeners can be supposed to constitute a background profile. Except the OctaCDD and 1,2,3,4,6,7,8-HeptaCDF, all of them have loadings higher than 0.3.

The loading value of OctaCDD is border-line, but this congener is usually determined in all tissues. This value could depend on steric hindrance associated with molecule size [4]. To our knowledge, the half-life of this congener has not been experimentally determined although it has been calculated using models. Much shorter halflives than usual have been reported for several PCDDs and PCDFs, including OctaCDD in breast-fed infants, but the data were calculated by models [5]. The congeners with the highest scores (Table 1) of the first PC appear to derive from general combustion source(s) [7], such as indicated previously. These congeners include $1,2,3,7,8-\mathrm{PeCDD}$, 1,2,3,4,7,8-HxCDD, 1,2,3,6,7,8-HxCDD, 1,2,3,4,6,7,8-HpCDD, 2,3,4,7,8-PeCDF, 1,2,3,4,7,8-HxCDF and 1,2,3,6,7,8-HxCDF. PC2, however, is dominated by $1,2,3,4,6,7,8-\mathrm{HeptaCDF}$, which appears to constitute an industrial fingerprint, suggesting that the database contains samples with a specific contamination source: these samples account for a quotient of variance of $11.1 \%$. To define a background profile, the variability of the database should be reduced because the subjects should be not exposed to certain sources such as specific foods with a high lipid content and/or oily fish. Lifestyle variations, such as the use of pharmaceuticals [8], can affect the bioavailability of such compounds and therefore influence the determination of a background

\begin{tabular}{|l|l|l|}
\hline \multicolumn{1}{|c|}{} & PC1 & PC2 \\
\hline eigenvalues & 6.7 & 1.1 \\
\hline percent of variance (\%) & 66.7 & 11.1 \\
\hline cumulative percentage (\%) & 66.7 & 78.02 \\
\hline Log[pg1,2,3,7,8-PeCDD/g lb] & 0.316 & -0.150 \\
\hline Log[pg 1,2,3,4,7,8-HxCDD)/g lb] & 0.327 & -0.245 \\
\hline Log[pg 1,2,3,6,7,8-HxCDD/g lb] & 0.350 & -0.236 \\
\hline Log[pg 1,2,3,7,8,9-HxCDD/g lb] & 0.310 & -0.178 \\
\hline Log[pg 1,2,3,4,6,7,8-HpCDD/g lb] & 0.333 & 0.153 \\
\hline Log[pg OCDD/g lb] & 0.297 & 0.009 \\
\hline Log[pg 2,3,4,7,8-PeCDF)/g Ib] & 0.349 & -0.073 \\
\hline Log[pg 1,2,3,4,7,8-HxCDF)/g lb] & 0.333 & 0.223 \\
\hline Log[pg 1,2,3,6,7,8-HxCDF)/g lb] & 0.358 & 0.167 \\
\hline Log[pg 1,2,3,4,6,7,8-HpCDF)/g lb] & 0.121 & 0.850 \\
\hline
\end{tabular}

Table 1: Principal Component (PC1 and $\mathrm{PC} 2)$ loadings with variance quote estimations.

*Corresponding author: Roberto Miniero, Chemical Toxicology Unit, Department of the Environment and Primary Intervention, Istituto Superiore di Sanità, Viale Regina Elena 299, 00161 Rome, Italy, Tel: +39 06 49902067; Fax: +39 06 49387139; E-mail: roberto.miniero@iss.it

Received July 26, 2013; Accepted October 28, 2013; Published November 04 2013

Citation: Miniero R, Felip ED, di Domenico A (2013) Suggestions for the Preliminary Definition of a Current Background Profile of Polychlorodibenzodioxin (PCDDs) and Polychlorodibenzofuran (PCDFs) Congeners in Serum Samples. J Bioequiv Availab 5: 242-243. doi:10.4172/jbb.1000166

Copyright: @ 2013 Miniero R, et al. This is an open-access article distributed under the terms of the Creative Commons Attribution License, which permits unrestricted use, distribution, and reproduction in any medium, provided the original author and source are credited. 
profile. However, the samples in Figure 1 (sample scores) are clustered around the centre of the graph, indicating low variability among the samples. This method could be improved by including a further selection of data covering all the factors potentially involved, including latent, exogenous and endogenous variables.

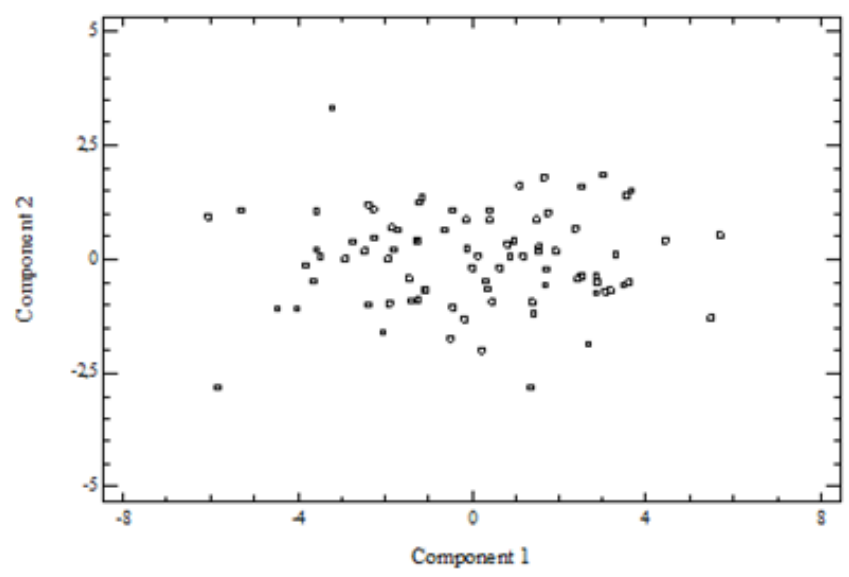

Figure 1: Scatterplot of sample scores.

\section{References}

1. Sun SJ, Zhao JH, Liu HJ, Liu DW, Ma YX, et al. (2006) Dioxin concentration in human milk in Hebei Province and Tokio, Japan: Potentially dietary risk factors and determination of possible sources. Chemosphere 62: 1879-1888.

2. www.unep.org

3. Schecter (2012) Dioxin and Health, John Wiley \& Sons, Inc., New Jersey (USA), 659.

4. Fielding $\mathrm{AH}$ (2007) Cluster and Classification Techniques for the biosciences Cambridge University Press $245 \mathrm{pp}$

5. Bruggeman WA, Opperhuizen A, Wijbenga O, Hutzinger O (1984) Bioaccumulation of super-lipophilic chemicals in fish. Toxicological and Environmental Chemistry 7: 173-189.

6. Leung HW, Kerger BD, Paustenbach DJ (2006) Elimination half-lives of selected polychlorinated dibenzodioxins and dibenzofurans in breast-fed human infants. J Toxicol Environ Health A 69: 437-443.

7. Hutzinger $\mathrm{O}$ and Fiedler H (1993) From sources to exposure: some open questions. Chemosphere 27: 121-129.

8. Zeliger HI (2011) Human toxicology of chemical mixtures, II Ed. Elsevier, United Kingdom, 575. 